\title{
Exhaled nitric oxide and asthma control: a longitudinal study in unselected patients
}

\author{
A. Michils, S. Baldassarre and A. Van Muylem
}

ABSTRACT: Controlled studies have shown that monitoring of the exhaled nitric oxide fraction ( $F$ eNO) improves asthma management. However, the studies seldom consider the full range of patients seen in clinical practise. In the present study, the ability of $F$ eNO to reflect asthma control over time is investigated in a regular clinical setting, and meaningful FeNO cut-off points and changes are identified.

Answers to the Asthma Control Questionnaire and FeNO were recorded at least once in 341 unselected asthma patients. The whole population and subgroups were considered, i.e. both inhaled corticosteroid (ICS)-naïve and low or high-to-medium ( $\leqslant$ or $>500 \mu \mathrm{g}$ beclomethasone dipropionate equivalents $\cdot$ day $^{-1}$ ) ICS-dose groups.

An FeNO decrease $<\mathbf{4 0} \%$ or increase $<\mathbf{3 0} \%$ precludes asthma control optimisation or deterioration, respectively (negative predictive value 79 and $82 \%$, respectively). In the present study's low-dose group, a decrease $>40 \%$ indicated asthma control optimisation (positive predictive value (PPV) $83 \%$ ). In ICS-naïve patients, FeNO $>35 \mathrm{ppb}$ predicted asthma control improvement in response to ICS (PPV 68\%). In most cases, forced expiratory volume in one second assessments were not useful.

In conclusion, in a given patient, exhaled nitric oxide fraction was found to be significantly related to asthma control over time. The overall ability of exhaled nitric oxide fraction to reflect asthma control was reduced in patients using high doses of inhaled corticosteroids. Forced expiratory volume in one second had little additional value in assessing asthma control.

KEYWORDS: Asthma control, exhaled nitric oxide, lung function

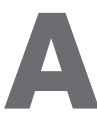
sthma control is a major goal of asthma management [1]. However, asthma is a complex syndrome with several phenotypic elements, such as airway inflammation, airway calibre, bronchial responsiveness and airway remodelling. Therefore, accurate assessment of asthma control may require a multidimensional approach that incorporates distinct parameters, such as symptoms, lung function and biomarkers. Randomised trials have recently shown that asthma management that considered inflammatory markers, such as sputum eosinophils and airway hyperresponsiveness, as a surrogate for inflammation resulted in improved asthma control [2, 3]. The degree of airway inflammation may also be reflected by the level of exhaled nitric oxide fraction $(F \mathrm{eNO})$, which is elevated in steroid-naïve asthma [4]. Further increases are seen during asthma exacerbations [5], whereas decreases occur after treatment with inhaled corticosteroids (ICS) [6]. A first longitudinal study by JONES et al. [7] showed the usefulness of FeNO monitoring for predicting and diagnosing loss of asthma control. Furthermore, a 1-yr follow-up, randomised study caused increased interest in monitoring FeNO in asthma patients by demonstrating that FeNO-guided asthma therapy resulted in the reduction of ICS doses without compromising asthma control [8]. Although a more recent study may slightly temper this enthusiasm [9], all these data suggest that FeNO may be a valuable indicator in the longitudinal assessment of asthma control. However, as with most controlled trials, each of these trials involved only selected patients who do not necessarily represent the full range of clinical situations [10]. In addition, several questions regarding the application of FeNO monitoring in the day-to-day management of asthma still remain to be resolved, including the issue of clinically meaningful FeNO cut-off points and changes [11].
AFFILIATIONS

Chest Dept, Erasme University Hospital, Brussels, Belgium.

CORRESPONDENCE

A. Van Muylem

Chest Dept

CUB Erasme

808 Route de Lennik

B-1070 Brussels

Belgium

Fax: 3225554411

E-mail: avmuylem@ulb.ac.be

Received:

February 192007

Accepted after revision:

October 312007

STATEMENT OF INTEREST

A statement of interest for this study can be found at

www.erj.ersjournals.com/misc/

statements.shtml 
The current authors therefore purposely performed a study that documents FeNO cut-off values and changes that can be considered clinically important in the longitudinal assessment of asthma control in a population of unselected asthma patients.

To do this, FeNO was monitored on several occasions in patients attending a tertiary asthma clinic. Its ability to reflect and to predict either improvement or worsening of asthma control over time was evaluated and compared with that of forced expiratory volume in one second (FEV1), using the Asthma Control Questionnaire (ACQ) [12] as a gold standard for the assessment of asthma control.

\section{METHODS}

\section{Subjects}

Between January 1, 2004 and April 30, 2007, 341 adult patients (164 males, mean age \pm SD $41 \pm 16$ yrs) attending the Allergy and Asthma Clinic in the Chest Dept of Erasme University Hospital (Brussels, Belgium) for treatment of persistent asthma diagnosed according to standard criteria [1] were prospectively enrolled in the present study. A total of 142 patients were newly diagnosed and had not received any specific treatment for asthma prior to inclusion. The remaining 199 patients regularly attended the outpatient clinic for treatment of chronic asthma and had already being given ICS either with or without other asthma medications (e.g. long-acting $\beta_{2^{-}}$ agonists $(n=157)$, leukotriene antagonists $(n=59)$, theophylline $(n=27)$, systemic steroids $(n=16)$, omalizumab $(n=6))$ in accordance with the recommendations of the international guidelines [1].

As the study was conducted in a regular clinical context, all patients with a definite diagnosis of asthma were included, with the exception of smokers because it has been shown that FeNO is suppressed by tobacco smoking [13]. Furthermore, quite unexpectedly, only $10 \%$ of the present patients were active smokers.

A total of $301(88 \%)$ patients were found to be allergic. The allergic status was evaluated using skin prick test or radioallergosorbent test against common inhalant allergens. Patients were asked not to use $\beta_{2}$-agonists $6 \mathrm{~h}$ prior to visits to the clinic.

The present study was approved by the local ethics committee and patients signed an informed consent form.

\section{Study procedures and design}

\section{Study design}

The study was designed as a prospective trial with post hoc data analysis. ACQ scores, FeNO and pre-bronchodilator FEV1 were recorded independently on one or several occasions for each patient. At each visit, asthma treatment was adjusted according to the recommendations of the Global Initiative for Asthma guidelines recommendations, regardless of the ACQ score or FeNO value, which were recorded separately.

Since the ultimate goal of asthma management is to achieve well-controlled asthma, the 0.75 optimum cut-off point [14] was selected as the reference ACQ score in the receiver operating characteristic (ROC) curve analysis, except when considering severe asthma. Using this technique, the abilities of FeNO and FEV1 to assess the following were studied: 1) to reflect asthma control as defined by an ACQ score $<0.75$ or $>0.75 ; 2)$ to detect and predict optimisation of asthma control defined as a minimum 0.5 change that allowed the ACQ score to decrease from $>0.75$ (not well-controlled asthma) to $<0.75$ (well-controlled asthma); and 3) detect and predict loss of optimal control defined as a minimum 0.5 change that allowed the ACQ score to increase from $<0.75$ (well-controlled asthma) to $>0.75$ (not well-controlled asthma).

Patients treated with low $(\leqslant 500 \mu \mathrm{g}$ beclomethasone dipropionate equivalents (BDPeq) $\cdot$ day $^{-1}$ ) and high-to-medium $\left(>500 \mu \mathrm{g} \mathrm{BDPeq} \cdot \mathrm{day}^{-1}\right.$ ) ICS doses were considered separately. Indeed, it has been shown that for ICS doses $<500 \mu \mathrm{g}$ BDPeq $\cdot$ day $^{-1}$, the relationship between FeNO and the antiinflammatory effect of ICS is linear, while at doses of $>500 \mu \mathrm{g}$ BDPeq $\cdot$ day $^{-1}$ FeNO levels may be low despite ongoing airway inflammation $[15,16]$. In the latter group (i.e. high-to-medium ICS doses), patients suffering from severe asthma, as defined according the American Thoracic Society (ATS) working group criteria [17], were considered separately. In this group, the FeNO ability to detect a significant change in asthma control was assessed (i.e. improvement (delta ACQ score >-0.5) was assessed rather than optimisation (ACQ score <0.75) and worsening (delta ACQ score $>+0.5$ ) rather than loss of optimal control). Indeed, it seemed unreasonable to expect severe asthmatics to achieve the same extent of control as moderateto-mild asthmatics.

\section{Study procedures}

Asthma Control Questionnaire

Asthma control was assessed using a French translation of the short version [18] of the ACQ taken from JUNIPER et al. [12]. This version does not include FEV1 rating. Patients subjectively evaluated the degree of impairment caused by their asthma during the preceding 7 days by responding to six questions using a seven-point scale; a score of 0 indicates no impairment and a score of 6 indicates maximal impairment. The total ACQ score is the mean of the six responses, varying between 0 (totally controlled asthma) and 6 (severely uncontrolled asthma). A recent analysis showed that the optimal cut-off point to identify a patient whose asthma is well controlled is 0.75 (i.e. if a patient has a score $\leqslant 0.75$, there is an $85 \%$ chance that their asthma is well controlled) [14]. In addition, a 0.5 change in the ACQ score is considered to be the minimum change that is clinically relevant [14].

\section{Exhaled nitric oxide fraction}

FeNO was measured before any forced expiratory manoeuvres using a daily calibrated LR 2000 chemoluminescence analyser (Logan Research Ltd, Rochester, UK) with on-line measurement of a single exhalation at flow rate of $50 \mathrm{~mL} \cdot \mathrm{s}^{-1}$ (ATS/ European Respiratory Society standard) [19]. Exhaled nitric oxide levels were read at the plateau corresponding to $70-80 \%$ of the carbon dioxide curve. Absolute FeNO values are expressed in $\mathrm{ppb}$, and changes in FeNO are expressed as a percentage of the initial value $(\Delta \%)$.

\section{Lung function}

Spirometry was performed using a Zan 300 spirometer (Zan ${ }$, Oberthulba, Germany). Pre-bronchodilator FEV1 was used as 
an index of airway calibre. FEV1 values are expressed as a percentage of predicted value (\% pred) [20], and changes in FEV1 are expressed as a percentage of the initial value $(\Delta \%)$.

\section{Statistical methods}

ROC curve analysis was performed in the whole population, as well as in the following different subgroups: steroid-naïve patients, patients treated by low and high-to-medium doses, and severe asthmatics. The area under the ROC curve (AUC) was computed and its difference from 0.5 was statistically evaluated. For a given type of assessment, the optimal cut-off value was determined for the whole population by maximising the Youden's index [21], i.e. the true positive rate (sensitivity) minus the false positive rate (1-specificity). This cut-off value was then used to derive sensitivity, specificity, and positive and negative predictive values in the whole population and in the subgroups of patients.

Unpaired t-tests were used when considering FEV1 and logtransformed FeNO values and Mann-Whitney U-tests when considering ICS doses and ACQ scores. The limit of significance was taken as 0.05 .

\section{RESULTS}

Table 1 presents FeNO, FEV1 and ACQ score values at study onset for the whole population $(n=341)$, for ICS-naïve patients, for patients treated with low ICS or high-to-medium ICS dose (excluding severe asthmatics), and for patients with severe asthma, respectively.

A total of 234 patients out of 341 were seen at least twice, representing 502 pairs of successive visits (median time between two visits: 80 days, range 10-1,129 days, interquartile interval 42-180). For nonsevere asthmatics, asthma was not well controlled (ACQ score $\geqslant 0.75$ ) at the first visit on 251 occasions and well-controlled on 164 occasions. In total, 87 pairs of visits involved patients with severe asthma that was not well controlled at the first visit on 73 occasions.

Tables 2-4 display the cut-off values resulting from Youden's index maximisation (see supplementary material), the number of positive and total cases and, therefore, the prevalence, the sensitivity (Se), the specificity (Sp), the positive (PPV) and negative (NPV) predictive values and the p-value allowing rejection (or not) of the null hypothesis (AUC=0.5). In the supplementary material, Se, Sp, PPV, NPV and accuracy may be found for other cut-off values, as well as the amounts of true-positive, true-negative, false-positive and false-negative cases (contingency tables). The way to derive PPV and NPV, given Se and $\mathrm{Sp}$, for any given prevalence (Baye's formulas) may also be found in the supplementary material.

\section{Cross-sectional assessment of asthma control}

Asthma control was assessed transversally at study onset for 324 nonsevere asthma patients. Well-controlled asthma (ACQ score $<0.75$ ) was considered as a positive event. Table 2 shows that in the whole population $(\mathrm{n}=324)$, FeNO level $>45 \mathrm{ppb}$ or FEV1 $<85 \%$ pred makes it possible to exclude a well-controlled asthma (NPV 89 and $80 \%$, respectively). It must be noted that FeNO operating characteristics in asthma control assessment worsen from ICS naïve to medium-to-high ICS-dose groups. FEV1 exhibits poor operating characteristics in assessing transversally asthma control.

\section{Change in asthma control between pairs of visits: assessment and prediction}

Optimisation and improvement assessment

In nonsevere asthma, disease control was not optimal at the first visit in 251 pairs (out of 415). Optimisation of asthma control (spontaneous as well as treatment induced) at visit two is considered as a positive event; this occurred on 92 occasions.

Table 3 shows that FeNO exhibits good operating characteristics, especially in the low ICS dose group: with a cut-off value at a $40 \%$ decrease, a high NPV is observed in all groups of patients. In the group of patients treated with low ICS dose, a high positive predictive value $(83 \%)$ is also found. Figure 1 illustrates the FeNO and FEV1 ROC curves for the total population, for patients with low ICS dose, and for patients with high-to-medium ICS dose.

Among the 73 pairs of visits involving patients with severe and uncontrolled asthma, an improvement in asthma control (i.e. a positive event) occurred on 32 occasions. FEV1 and FeNO show similar operating characteristics to detect such a change: an FeNO decrease $<15 \%$ or a FEV1 increase $<5 \%$ virtually

TABLE 1 Exhaled nitric oxide fraction (FeNO), forced expiratory volume in one second (FEV1) and Asthma Control Questionnaire (ACQ) score values at study onset

$\begin{array}{cccccc}\text { Subjects } & \text { FeNo ppb geometrical mean } \\ \text { (geometrical interval) } & \text { p-value } & \text { FEV } 1 \% \text { pred } & \text { p-value } & \text { ACQ score } & \text { p-value }\end{array}$

\begin{tabular}{|c|c|c|c|c|c|c|c|}
\hline Total & 341 & $32.9(13-8-78.1)$ & & $86.3 \pm 18.5$ & & $1.5(0-5.2)$ & \\
\hline ICS naïve & 142 & $49.8(24.0-103.5)$ & & $88.9 \pm 17.1$ & & $2.0(0-5.2)$ & \\
\hline $\begin{array}{c}\text { ICS dose } \leqslant 500 \mu \mathrm{g} \\
\text { BDPeq } \cdot \text { day }^{-1}\end{array}$ & 102 & $27.0(11.7-62.1)$ & $<0.001$ & $90.1 \pm 15.1$ & 0.59 & $0.8(0-4.8)$ & $<0.001$ \\
\hline $\begin{array}{c}\text { ICS dose }>500 \mu \mathrm{g} \\
\text { BDPeq }^{\text {day }^{-1}}\end{array}$ & 80 & $20.5(9.0-46.7)$ & $<0.001$ & $84.1 \pm 19.0$ & 0.034 & $1.3(0-5.2)$ & 0.002 \\
\hline Severe asthma & 17 & $31.3(15.9-62.1)$ & 0.014 & $52.8 \pm 11.4$ & $<0.001$ & $3.5(0.8-4.7)$ & 0.007 \\
\hline
\end{tabular}


TABLE 2 Cross-sectional assessment of asthma control

\begin{tabular}{|c|c|c|c|c|c|c|c|c|}
\hline & Total events $n$ & Positive cases $\mathbf{n}$ & Prevalence & $\mathrm{Se}$ & Sp & PPV & NPV & p-value ${ }^{\#}$ \\
\hline \multicolumn{9}{|l|}{ FeNO 45 ppb } \\
\hline ICS dose $0 \mu \mathrm{g} \mathrm{BDPeq} \cdot$ day $^{-1}$ & 142 & 17 & 12 & 59 & 67 & 20 & 92 & 0.039 \\
\hline ICS dose $\leqslant 500 \mu \mathrm{g}$ BDPeq $\cdot$ day $^{-1}$ & 102 & 46 & 45 & 87 & 34 & 52 & 76 & 0.036 \\
\hline ICS dose $>500 \mu \mathrm{g}$ BDPeq $\cdot$ day $^{-1}$ & 80 & 29 & 36 & 93 & 22 & 40 & 85 & 0.84 \\
\hline ICS dose $0 \mu \mathrm{g} \mathrm{BDPeq} \cdot$ day $^{-1}$ & 142 & 17 & 12 & 71 & 38 & 13 & 91 & 0.37 \\
\hline ICS dose $\leqslant 500 \mu \mathrm{g} \mathrm{BDPeq} \cdot$ day $^{-1}$ & 102 & 46 & 45 & 80 & 45 & 54 & 74 & 0.22 \\
\hline ICS dose $>500 \mu \mathrm{g} \mathrm{BDPeq} \cdot$ day $^{-1}$ & 80 & 29 & 36 & 64 & 52 & 44 & 73 & 0.42 \\
\hline
\end{tabular}

Data are presented as \%, unless otherwise indicated. Se: sensitivity; Sp: specificity; PPV: positive predictive value; NPV: negative predictive value; FeNO: exhaled nitric oxide fraction; ICS: inhaled corticosteroid; BDPeq: beclomethasone dipropionate equivalents; FEV1: forced expiratory volume in one second; \% pred: \% predicted \#: $p$-values represent the statistical significance of rejecting the area under the curve $=0.5$; ${ }^{\circ}$ : cut-off value. A positive event is a well-controlled asthma. A true positive case is defined as $\mathrm{FeNO}_{\mathrm{NO}} \leqslant 45 \mathrm{ppb}$ or FEV $1 \geqslant 85 \%$ pred associated with a well-controlled asthma.

excludes an improvement in asthma control (NPV 76 and 74\%, respectively; table 3 ).

Loss of optimal control and control worsening: assessment In nonsevere asthma, an optimal control was documented at the first visit in 164 pairs (out of 415). Loss of optimal control at visit two is considered as a positive event. This occurred in 39 occasions. Table 4 shows that, in the whole population, an FeNO increase $<30 \%$ makes a loss of optimal control unlikely (NPV 82\%). Nevertheless, in the high-to-medium ICS dose group, FeNO ability is lost. FEV1 has poor operating characteristics in all groups of patients, especially in the low ICS dose group.
In severe asthma, a worsening of asthma control (i.e. positive event) occurred on 25 occasions (out of 87 pairs). FEV1 and FeNO exhibit equivalent operating characteristics. An FeNO increase $<15 \%$ or an FEV1 decrease $<5 \%$ make a worsening of asthma control unlikely (NPV 78 and $81 \%$, respectively).

\section{Optimisation: prediction}

The present analysis was restricted to pairs of visits for which asthma was initially not well controlled and for which an antiinflammatory treatment was either started or increased.

In nonsevere asthma, 148 pairs of visits fulfilled these conditions and asthma control optimisation occurred in 65 occasions (i.e. positive event). The dose increments between the

\section{TABLE 3 Assessment of an optimisation or an improvement of asthma control}

\begin{tabular}{|c|c|c|c|c|c|c|c|c|}
\hline & Total events $\mathbf{n}$ & Positive cases $n$ & Prevalence & $\mathrm{Se}$ & $\mathrm{Sp}$ & PPV & NPV & p-value \\
\hline \multicolumn{9}{|l|}{ Optimisation } \\
\hline Total & 251 & 92 & 37 & 64 & 78 & 63 & 79 & $<0.001$ \\
\hline ICS dose $\leqslant 500 \mu \mathrm{g} \mathrm{BDPeq} \cdot$ day $^{-1}$ & 106 & 56 & 53 & 70 & 84 & 83 & 71 & $<0.001$ \\
\hline ICS dose $>500 \mu \mathrm{g} \mathrm{BDPeq} \cdot$ day $^{-1}$ & 145 & 36 & 25 & 53 & 75 & 41 & 83 & $<0.001$ \\
\hline ICS dose $\leqslant 500 \mu \mathrm{g} \mathrm{BDPeq} \cdot$ day $^{-1}$ & 106 & 56 & 53 & 34 & 70 & 56 & 49 & 0.21 \\
\hline ICS dose $>500 \mu \mathrm{g} \mathrm{BDPeq} \cdot$ day $^{-1}$ & 145 & 36 & 25 & 61 & 69 & 39 & 84 & $<0.001$ \\
\hline \multicolumn{9}{|l|}{ Improvement $^{\S}$} \\
\hline FeNO $-15 \%^{+}$ & 73 & 32 & 44 & 72 & 70 & 66 & 76 & $<0.001$ \\
\hline $\mathrm{FEV}_{1}+5 \%^{+}$ & 73 & 32 & 44 & 72 & 62 & 59 & 74 & $<0.001$ \\
\hline
\end{tabular}

Data are presented as \%, unless otherwise indicated. Se: sensitivity; Sp: specificity; PPV: positive predictive value; NPV: negative predictive value; FeNO: exhaled nitric oxide fraction; ICS; inhaled corticosteroid; BDPeq: beclomethasone dipropionate equivalents; FEV1: forced expiratory volume in one second. ${ }^{\#}$ : p-values represent the statistical significance of rejecting the area under the curve $=0.5 ;{ }^{*}$ : nonsevere asthma; ${ }^{+}$: cut-off value; ${ }^{\text {s: }}$ : severe asthma. In nonsevere asthma, a positive event is defined as an optimisation of asthma control. A true positive event is defined as an $\mathrm{FeNO}$ change $\leqslant-40 \%$ (e.g. $-45 \%$ ) or an FEV 1 change $\geqslant 5 \%$ associated with an optimisation of asthma control between consecutive visits. In severe asthma, a positive event is defined as an improvement of asthma control. A true positive case is defined as an FeNO change $\leqslant-15 \%$ (e.g. $-20 \%$ ) or an $\mathrm{FEV} 1$ change $\geqslant 5 \%$ associated with an improvement of asthma control between consecutive visits. 
TABLE 4 Assessment of a loss of optimal asthma control or a worsening of asthma control

\begin{tabular}{|c|c|c|c|c|c|c|c|c|}
\hline & Total events $n$ & Positive cases $\mathrm{n}$ & Prevalence & $\mathrm{Se}$ & $\mathrm{Sp}$ & PPV & NPV & p-value ${ }^{\#}$ \\
\hline \multicolumn{9}{|l|}{ Loss of optimal control } \\
\hline Total & 164 & 39 & 24 & 54 & 66 & 33 & 82 & 0.021 \\
\hline ICS dose $\leqslant 500 \mu \mathrm{g} \mathrm{BDPeq} \cdot$ day $^{-1}$ & 104 & 19 & 18 & 74 & 64 & 31 & 92 & 0.002 \\
\hline ICS dose $>500 \mu \mathrm{g}$ BDPeq $\cdot$ day $^{-1}$ & 60 & 20 & 33 & 35 & 70 & 37 & 68 & 0.39 \\
\hline ICS dose $\leqslant 500 \mu \mathrm{g} \mathrm{BDPeq} \cdot$ day $^{-1}$ & 104 & 19 & 18 & 21 & 91 & 33 & 84 & 0.51 \\
\hline ICS dose $>500 \mu \mathrm{g}$ BDPeq $\cdot$ day $^{-1}$ & 60 & 20 & 33 & 45 & 88 & 64 & 76 & 0.062 \\
\hline \multicolumn{9}{|l|}{ Worsening $^{\S}$} \\
\hline $\mathrm{FeNO}+15 \%^{+}$ & 87 & 25 & 29 & 52 & 67 & 39 & 78 & 0.008 \\
\hline FEV $1-5 \%^{+}$ & 87 & 25 & 29 & 57 & 78 & 50 & 81 & $<0.001$ \\
\hline
\end{tabular}

Data are presented as \%, unless otherwise indicated. Se: sensitivity; Sp: specificity; PPV: positive predictive value; NPV: negative predictive value; FeNO: exhaled nitric oxide fraction; ICS: inhaled corticosteroid; BDPeq: beclomethasone dipropionate equivalents; FEV1: forced expiratory volume in one second. ${ }^{*}$ : p-values represent the statistical significance of rejecting the area under the curve $=0.5 ;{ }^{\uparrow}$ : nonsevere asthma; ${ }^{+}$: cut-off value; ${ }^{\S}$ : severe asthma. In nonsevere asthma, a positive event is defined as a loss of optimal asthma control. A true positive event is defined as an FeNO change $\geqslant 30 \%$ or an FEV 1 change $\leqslant-10 \%$ (e.g. $-15 \%$ ) associated with a loss of optimal asthma control between consecutive visits. In severe asthma, a positive event is defined as a worsening of asthma control. A true positive case is defined as an FeNO change $\geqslant 15 \%$ or an FEV1 change $\leqslant-5 \%$ (e.g. $-10 \%)$ associated with a worsening of asthma control between consecutive visits.

two visits (mean $523 \mu \mathrm{g}$ BDPeq·day ${ }^{-1}$ ) were not different in patients who did or did not exhibit asthma control optimisation $(p=0.39)$. For those patients already treated with ICS at the


similar in the two groups $(\mathrm{p}=0.19)$.

In steroid naïve patients, an initial $F$ eNO level $>35$ ppb predicts asthma control optimisation in two out of three cases (PPV $68 \%$ ). In ICS-treated patients, asthma control is unlikely to become optimal after treatment increase if FeNO was $<35 \mathrm{ppb}$ at the first visit (NPV 88\%). FEV1 never predicted optimisation. For details of the results for several cut-off points please refer to the supplementary material (table 7).

In the population of severe asthmatics, 27 pairs of visits fulfilled the conditions (asthma not well-controlled at first visit and treatment increase), and an improvement was seen on 14 occasions. However, if the anti-inflammatory treatment was similar in patients who did or did not improve their asthma control, the increase in treatment in systemic corticoids was significantly higher in the group exhibiting an improvement $(p=0.006)$. Given this bias, improvement prediction was not considered in this group of patients.

\section{Loss of optimal control: prediction}

In the population of nonsevere asthmatics, this analysis was restricted to pairs of visits for which asthma was initially well controlled and for which visits were separated by no more than 3 months. In total, 61 pairs fulfilled these conditions and only 11 pairs exhibited a loss of optimal control at the subsequent visit. No difference appeared with regard to either initial ICS dosage or in treatment modification between patients who did or did not show a loss of optimal asthma control $(\mathrm{p}=0.98$ and $\mathrm{p}=0.61$, respectively). An FeNO level $<30 \mathrm{ppb}$, along with well-controlled asthma, indicates that a loss of optimal control is unlikely to occur within the next
3 months (NPV 94\%). FEV1 has no predictive power. For details of the results for several cut-off points please refer to the supplementary material (table 8).

In the group of severe asthmatics, 49 pairs of visits fulfilled the conditions and on only 11 occasions was a worsening of asthma control observed at the subsequent visit. However, if the anti-inflammatory treatment was similar in patients who did or did not worsen their asthma control, a treatment increment in systemic corticoids was significantly higher in the group without worsening $(\mathrm{p}=0.027)$. Given this bias, worsening prediction was not considered in the group of severe asthmatics.

\section{DISCUSSION}

The present study shows that FeNO is a reliable marker of asthma control in unselected asthma patients, especially in those patients treated with low doses of ICS. The current data also indicate that changes in FeNO values, rather than absolute cut-off points (i.e. personalised FeNO profiles), may be meaningful for the longitudinal assessment of asthma control.

When the isolated assessment of asthma control was first considered (table 2), the present authors found that an FeNO level $>45 \mathrm{ppb}$ indicated that asthma was not well controlled, but only for steroid-naïve and low ICS dose-treated patients. This is in accordance with the correlation recently documented between FeNO and asthma control in newly diagnosed asthmatics, which used another questionnaire, the Asthma Control Test, albeit well correlated to the ACQ [14], to assess asthma control [22]. When considered patients were treated with high-to-medium ICS doses, Fe,NO no longer had the ability to reflect asthma control. This may partly explain the discrepancy with recently published data, which indicated no association between ACQ score and FeNO level in a study in which ICS-naïve and ICS-treated patients were pooled [23]. In 

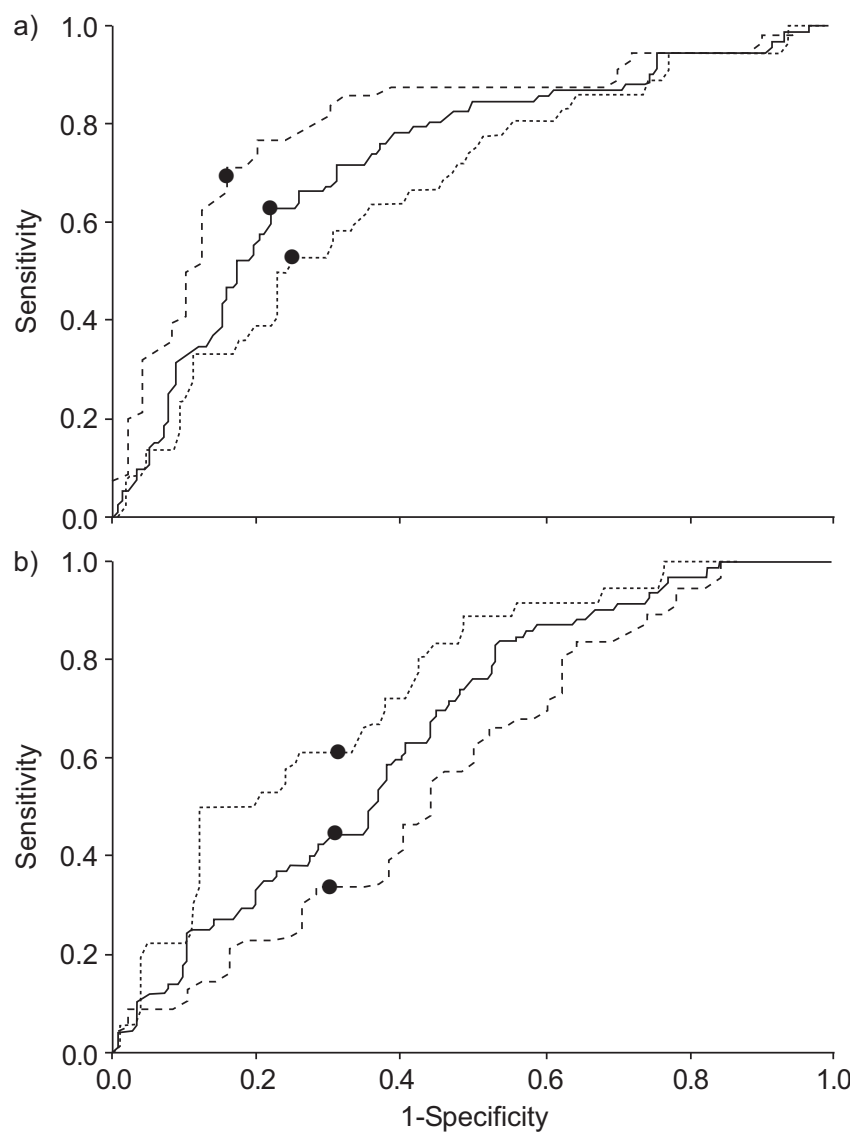

FIGURE 1. Receiver operating curves characterising the ability of a) exhaled nitric oxide fraction ( $\mathrm{FeNO}$ ) and b) forced expiratory volume in one second ( $\left.F E V_{1}\right)$ to assess an optimisation of asthma control in nonsevere asthma. — : whole population; -----: patients treated with low inhaled corticosteroid (ICS) dose; ......... patients treated with high-to-medium ICS dose. $\bullet$ : cut-off value of a) $-40 \%$ change in $\mathrm{FeNO}_{\mathrm{NO}}$ and b) $5 \%$ change in FEV 1 .

the current study, no association was documented between asthma control and single measurement of FEV1. This confirms previous data showing a poor relationship between airway obstruction and respiratory symptoms in adult asthmatics [24].

These results, taken together, suggest that isolated measurements of FeNO and FEV1 do not appear very successful in capturing asthma control. In fact, control instruments (i.e. questionnaires) may more accurately perceive changes in asthma symptoms rather than the asthma symptoms themselves.

However, asthma is a chronic disorder and long-term followup with repeated assessments of various parameters is required in order to make proper treatment adjustments. JONES et al. [7] were the first to tackle the issue of FeNO use in the longitudinal assessment of asthma control. Using a steroid withdrawal protocol to mimic exacerbations, JONES et al. [7] were able to show that changes in FeNO levels (i.e. $+60 \%$ ) were more useful than single cut-off points in predicting and diagnosing loss of asthma control. This finding is confirmed in the present study involving patients who experienced spontaneous deteriorations of asthma control. In this case, an
FeNO increase $<30 \%$ would be helpful to exclude the occurrence of a significant deterioration of asthma control (table 4). The discrepancies in FeNO changes documented in the two studies may be explained by differences in the study designs: treatment regimens (i.e. ICS withdrawal versus ICS maintenance) and study end-points (loss of control versus loss of optimal control) were different. The difference between the PPVs exhibited by FeNO in diagnosing loss of control in the two studies $(87 \%$ in the study by JONES et al. [7] versus $33 \%$ in the present study) is, at least partially, related to the differences in the prevalence of loss of control in each study (78\% in the study by JONES et al. [7] versus $24 \%$ in the present study). It must be noted, finally, that the ability of Fe,NO to detect deteriorating asthma was shown to be rather limited in other controlled trials using reduction or short withdrawal of ICS therapy $[25,26]$.

In addition, the present study indicates that sequential FeNO measurements may also be helpful with regard to indicating improvement in asthma control over time (table 3). Thus, when asthma is not optimally controlled, a $40 \%$ FeNO reduction is a reliable predictor of asthma control optimisation, particularly for those patients treated with low ICS doses (PPV 80\%). In the group of patients treated with ICS doses $>500 \mu \mathrm{g}$ BDPeq $\cdot$ day $^{-1}$ (almost always $\geqslant 1,000 \mu \mathrm{g}$ BDPeq $\cdot$ day $^{-1}$ in the present study), the ability of FeNO to reflect changes in asthma control was somewhat reduced, suggesting that ICS doses might have to be taken into account when using FeNO to assess asthma control. However, even in severe asthmatics treated with high-to-very high ICS doses, a 15\% FeNO change would still apparently be helpful to rule out a significant change in asthma control confirming the usefulness of FeNO assessment in this population [27].

Taken together, the present data suggest that personalised FeNO profiles may be meaningful for asthma follow-up in unselected patients, at least when considering asthma control as assessed by the ACQ over a 3-month period (the median time between two visits in the present study). However, it must be acknowledged that, due to small group sizes, the present study was unable to account for factors, such as smoking and atopy, which may have an effect on the relationship between FeNO and asthma control. So far, it is not known whether the use of FeNO profiles rather than absolute cut-off points would have provided different results in the two controlled trials that have investigated the impact of FeNO-guided therapy on asthma control over 1 yr $[8,9]$.

In addition, FeNO assessment also appears helpful to predict ICS responsiveness (see table 7, supplementary material). Established guidelines recommend the use of ICS as the firstline treatment for chronic asthma [1]. These recommendations are based on clinical studies showing the overall efficacy of ICS for treating asthma. However, heterogeneity in the response to ICS treatment has been reported [28]. In a randomised trial, SMITH et al. [29] have previously documented a $47 \mathrm{ppb}$ threshold value that proved to be a reliable predictor of ICS responsiveness in patients with undiagnosed respiratory symptoms. In the present study, involving patients with a definite diagnosis of asthma and focusing only on asthma control rather than on the various outcomes investigated in the study by SMITH et al. [29], a 35-ppb threshold value emerges as 
a helpful predictor of ICS responsiveness: two out of three steroid-naïve patients with an FeNO level $>35 \mathrm{ppb}$ have improved control over their asthma after starting a treatment that includes ICS. In those patients who were already treated but not yet optimally controlled, an improvement in asthma control resulting from an increase in ICS dose is much less likely to occur if the FeNO level is not $>35 \mathrm{ppb}$.

Finally, an FeNO level $<30 \mathrm{ppb}$ in stable treated patients predicts that an exacerbation is unlikely to occur within the next 3 months. The cut-off point is similar to that documented in a recent controlled trial involving a much longer follow-up period (i.e. 18 months) [30].

When lung function was considered, FEV1 values did not appear to adequately reflect improvement or worsening of asthma control in the group of patients treated with low ICS doses, and who exhibited near normal lung function. This did not hold completely true anymore for more severe asthmatic patients treated with high ICS doses; these patients had a more altered lung function that left more room for improvement. It might be concluded that the lack of control in steroid naïve and low ICS dose-treated patients is likely to depend on airway inflammation, while in more severe asthmatics treated with a high ICS dose it may be the impairment in airway calibre that is the main culprit of poor control.

In conclusion, in a given patient, the exhaled nitric oxide fraction is significantly related to asthma control over time. The overall ability of the exhaled nitric oxide fraction to reflect asthma control is reduced in patients using high doses of inhaled corticosteroids. Forced expiratory volume in one second has little additional value in assessing asthma control.

\section{ACKNOWLEDGEMENTS}

The authors would like to thank E. Juniper (McMaster University, Hamilton, ON, Canada) for kindly allowing them to use the Asthma Control Questionnaire. The authors also thank M. Demesmaeker, M. Danschutter and J. Storms (all Erasme University, Brussels, Belgium) for technical assistance, C. Piesen for typing the manuscript and D. Young (Young \& Associates Ltd, London, UK) for advice.

\section{REFERENCES}

1 Global initiative for Asthma. Global strategy for asthma management and prevention: NHLBI/WHO Workshop Report. Washington, DC, U.S. Government Printing Office, 2002. NHLBI Publication 02-3659.

2 Green RH, Brightling CE, McKenna S, et al. Asthma exacerbations and sputum eosinophil counts: a randomised controlled trial. Lancet 2002; 360: 1715-1721.

3 Sont JK, Willems LN, Bel EH, et al. Clinical control and histopathologic outcome of asthma when using airway hyperresponsiveness as an additional guide to long-term treatment. The AMPUL Study Group. Am J Respir Crit Care Med 1999; 159: 1043-1051.

4 Alving K, Weitzberg E, Lundberg JM. Increased amount of nitric oxide in exhaled air of asthmatics. Eur Respir J 1993; 6: 1368-1370.

5 Crater SE, Peters EJ, Martin ML, Andrew WM, PlattsMills TAE. Expired nitric oxide and airway obstruction in asthma patients with an acute exacerbation. Am Resp Crit Care Med 1999; 158: 806-811.

6 Kharitonov SA, Yates DH, Barnes PJ. Inhaled glucocorticoids decrease nitric oxide in exhaled air of asthmatic patients. Am J Respir Crit Care Med 1996; 153: 454-457.

7 Jones SL, Kittelson J, Cowan JO, et al. The predictive value of exhaled nitric oxide measurements in assessing changes in asthma control. Am J Respir Crit Care Med 2001; 164: 738-743.

8 Smith AD, Cowan JO, Brassett KP, Herbison GP, Taylor DR. Use of exhaled nitric oxide measurements to guide treatment in chronic asthma. N Engl J Med 2005; 352: 2163-2173.

9 Shaw DE, Berry MA, Thomas M, et al. The use of exhaled nitric oxide to guide asthma management. Am J Respir Crit Care Med 2007; 176: 231-237.

10 Travers J, Marsh S, Williams M, et al. External validity of randomised controlled trials in asthma: to whom do the results of the trials apply? Thorax 2007; 62: 219-223.

11 Taylor RD, Pijnenburg MW, Smith AD, De Jongste JC. Exhaled nitric oxide measurements: clinical application and interpretation. Thorax 2006; 61: 817-827.

12 Juniper EF, O’Byrne PM, Guyatt GH, Ferrie PJ, King DR. Development and validation of a questionnaire to measure asthma control. Eur Respir J 1999; 14: 902-907.

13 McSharry CP, McKay IC, Chaudhuri R, Livingston E, Fraser I, Thomson NC. Short and long-term effects of cigarette smoking independently influence exhaled nitric oxide concentration in asthma. J Allergy Clin Immunol 2005, 116: 88-93.

14 Juniper EF, Bousquet J, Abetz L, Bateman ED, the GOAL Committee. Identifying "well-controlled" and "not well controlled" asthma using the asthma control questionnaire. Respir Med 2006; 73: 296-305.

15 Jatakanon A, Kharitonov S, Lim S, Barnes PJ. Effect of differing doses of inhaled budesonide on markers of airway inflammation in patients with mild asthma. Thorax 1999; 54: 108-114.

16 Jones SL, Herbison P, Cowan JO, et al. Exhaled NO and assessment of anti-inflammatory effects of inhaled steroid: dose-response relationship. Eur Respir J 2002; 20: 601-608.

17 Wenzel S. Severe asthma in adults. Am J Resp Crit Care Med 2005; 172: 149-160.

18 Juniper EF, Svensson K, Mörk AC, Stahl E. Measurement properties and interpretation of three shortened versions of the asthma control questionnaire. Respir Med 2005; 99: 553-558.

19 American Thoracic Society, European Respiratory Society. ATS/ERS recommendations for standardized procedures for the online and offline measurement of exhaled lower respiratory nitric oxide and nasal nitric oxide, 2005. Am J Respir Crit Care Med 2005; 171: 912-930.

20 European Respiratory Society. Standardized lung function testing: lung volumes and forced ventilatory flows. Eur Respir J 1993; 6: Suppl. 16, 5-40.

21 Youden WJ. Index for rating diagnostic tests. Cancer 1950; 3: 32-35.

22 Senna G, Passalacqua G, Chiapoli M, Lombardi C, Wilcock L. Correlation among FEV1, nitric oxide and asthma control test in newly diagnosed asthma. Allergy 2007; 62: 207.

23 Lopes C. FeNO 35ppb - is it associated with asthma control? J Allergy Clin Immunol 2006; 117: Suppl. 2, S177. 
24 Teeter J, Bleecker E. Relationship between airway obstruction and respiratory symptoms in adult asthmatics. Chest 1998; 113: 272-277.

25 Jatakanon A, Lim S, Barnes P. Changes in sputum eosinophils predict loss of asthma control. Am J Resp Crit Care Med 2000; 161: 64-72.

26 Leuppi JD, Salome CM, Jenkins CR, et al. Predictive markers of asthma exacerbation during stepwise dose reduction of inhaled corticosteroids. Am J Respir Crit Care Med 2001; 163: 406-412.

27 Silkoff PE, Lent AM, Busacker AA, et al. Exhaled nitric oxide identifies the persistent eosinophilic phenotype in severe refractory asthma. J Allergy Clin Immunol 2005; 116 : 1249-1255.

28 Szefler SJ, Phillips BR, Martinez FD, et al. Characterization of within-subject responses to fluticasone and montelukast in childhood asthma. J Allergy Clin Immunol 2005; 115: 233-242.

29 Smith AD, Cowan JO, Brassett KP, et al. Exhaled nitric oxide: a predictor of steroid response. Am J Respir Crit Care Med 2005; 172: 453-459.

30 Gelb AF, Flynn Taylor C, Shinar CM, Gutierrez C, Zamel N. Role of spirometry and exhaled nitric oxide to predict exacerbations in treated asthmatics. Chest 2006; 129: 1492-1499. 